\title{
Percepción de riesgo de abuso sexual entre adolescentes escolarizados de la ciudad de Cali*
}

\author{
Janeth Mosquera, TS, M ag TS, M ag Epidemiol ${ }^{1}$, Amparo Bermúdez, Hist, Mag Edu ${ }^{2}$ \\ RESUMEN
}

Antecedentes: El abuso sexual es un hecho de alta incidencia que ocurre en distintos estratos socio-económicos, culturas, ámbitos y etapas de la vida. Sin embargo, se ha explorado poco la percepción de riesgo que sobre este suceso tienen los adolescentes, uno de los grupos poblacionales más comprometido.

Métodos: Como complemento de un estudio transversal en el que se identificaron tres zonas en Cali con mayor prevalencia de denuncias de abuso sexual en población de 10 a 19 años de edad, se realizó una exploración cualitativa con adolescentes escolarizados de esas zonas, para indagar aspectos relacionados con la percepción de riesgo de abuso sexual. Participaron 121 adolescentes (53 hombres y 68 mujeres). La información se colectó con la técnica de grupo focal.

Resultados: Los hallazgos principales del estudio muestran que tanto hombres como mujeres adolescentes conciben el abuso sexual como violación (penetración de pene u otro objeto dentro de la vagina o el ano) y, por tanto, es la modalidad que a su juicio reviste mayor gravedad, así como prevalece el estereotipo de abusador de sexo masculino. Adicionalmente, se observaron diferencias en la percepción de riesgo según el género, relacionadas con los espacios públicos y domésticos y con las actitudes de culpabilizar a la víctima.

Conclusiones: La investigación sugiere la necesidad que programas de prevención de abuso sexual en adolescentes, enfaticen en las habilidades para identificar situaciones que los ponen a riesgo de abuso sexual en cualquiera de sus modalidades. Además, esos programas deben tener perspectiva de género, con el fin de clarificar distintas percepciones de riesgo y actitudes hacia las víctimas.

Palabras clave: Abuso sexual; Violencia sexual; Percepción de riesgo; Adolescentes.

Risk perception of sexual abuse among young students in Cali

\section{SUMMARY}

Background: The incidence of sexual abuse is high and occurs in all socioeconomic strata, cultures, spaces, and during different life stages. However, very little research has been conducted to explore risk perception among adolescents, even though adolescents are more likely to experience sexual violence.

Methods: Complementary with a transversal study, which identified three zones in Cali with higher prevalence of sexual abuse reported among youngsters between 10 and 19 years of age, we carried out a qualitative study to explore risk perception of sexual abuse among young students. _In this study, 121 adolescents from both sexes participated. The information was gathered by using a focal group technique.

Results: Main study results show that women and men have a notion of sexual abuse only related to rape or sodomy (penetration of the penis or other object into the vagina or anus), and they consider this a more serious type of sexual abuse. In addition, we observed differences in risk perception related to gender and domestic and public spaces. Finally, victim-blame attitudes are prevalent and the stereotypical male image as rapist is predominant.

Conclusions: The research suggest that is necessary in sexual abuse prevention programmes with youngsters to

* Proyecto financiado por COLCIENCIAS bajo contrato RC N 379-2004, código 11060416452 con el Instituto CISALVA de la Universidad del Valle, Cali, Colombia.

1. Docente Hora Cátedra, Escuela de Salud Pública, Facultad de Salud, Universidad del Valle, Cali, Colombia.

e-mail: jamosquerabecerra@gmail.com

2. Profesora Asociada, Escuela de Salud Pública, Facultad de Salud, Universidad del Valle, Cali, Colombia.

e-mail:amparitob@gmail.com

Recibido para publicación marzo 30, 2009 Aceptado para publicación enero 12, 2010 
emphasize aspects related to being able to identify circumstances that place them at risk of sexual abuse in any modality. Furthermore, prevention programmes should have a gender perspective to clarify different risks perceptions and attitudes about victims.

Keywords: Sexual abuse; Sexual violence; Risk perception; Adolescents.

El abuso sexual caracterizado como un comportamiento sexual que ocurre sin el consentimiento de la persona o bajo la coacción de una de las partes y clasificado por la Organización Mundial de la Salud en el grupo de violencia interpersonal ${ }^{1}$, es un evento de alta incidencia que ocurre en distintos estratos socio-económicos, culturas, ámbitos y etapas de la vida.

Las mujeres son quienes más sufren sus impactos, con cifras de ocurrencia de este fenómeno que oscilan entre $2 \%$ y $62 \%^{2}$. Sin embargo, es necesario enfatizar que entre $5 \%$ y $10 \%$ de los hombres, han expresado haber sido también víctimas de abuso sexual en la etapa de la niñez ${ }^{1}$. Cabe considerar que debido a problemas relacionados con la definición usada (abuso sexual, violencia sexual, maltrato sexual, entre otras), la metodología de medición, la población participante y los aspectos morales y jurídicos relacionados con este fenómeno, las cifras de abuso sexual podrían ser superiores.

La gravedad de las consecuencias psicológicas y físicas de un abuso en la infancia o en la adolescencia está mediada por las características mismas del abuso sexual (por ejemplo, uso de la violencia, penetración o no penetración) y se relacionan con el establecimiento de conductas sexuales de riesgo (alto número de parejas sexuales, sexo sin protección) y mayores prevalencias de infección de transmisión sexual ${ }^{3}$.

Aunque se tiende a considerar que son las niñas (menores de 10 años de edad), quienes con más frecuencia se ven enfrentadas al abuso sexual, los datos muestran que son las adolescentes las que mayor padecen esta situación ${ }^{4}$ y se ha indicado que por lo menos la mitad de las victimas de incesto son mujeres adolescentes $^{5}$. En la investigación de Sugar et al. ${ }^{6}$, se indicó que del total de mujeres asistentes a un centro de atención de emergencias para casos de asalto sexual, $23.1 \%$ de los casos correspondieron a adolescentes mujeres y la mayor parte de todas las mujeres (62\%) fueron víctimas de amigos o conocidos.
Particularmente, en Colombia para el año 2005 se encontró una tasa de 40.1 casos de delitos sexuales por cada 100,000 habitantes, ocupando este evento el $4^{\circ}$ lugar dentro del conjunto de lesiones violentas no fatales; siendo los menores de 18 años de edadel grupo más afectado $(70.6 \%)$ y, en dicho grupo, la población de 10 a 14 años presenta la tasa más alta (103.6 por 100,000$)$, seguida del grupo de los 5 a 9 años (82 por 100,000) y, posteriormente, de los adolescentes de 15 a 17 años $(67.4 \text { por } 100,000)^{7}$.

Como se tiene en cuenta la magnitud de la ocurrencia del abuso sexual en el ámbito mundial y nacional y y como se consideran las secuelas psicológicas y físicas a corto, mediano y largo plazo de este fenómeno, se hizo un estudio con enfoques cualitativo y cuantitativo,para identificar las características del abuso sexual en la población entre 10 y 19 años de edad en Cali ${ }^{8}$.

Los resultados del estudio cuantitativo ${ }^{9}$, queincluyó la geo-referenciación de los casos de denuncia de delitos sexuales durante el período 2001-2003, posibilitaron identificar las comunas de la ciudad de Cali con mayor prevalencia de informe de casos. Uno de los componentes cualitativos, correspondió a explorar la percepción de riesgo de abuso sexual en adolescentes escolarizados de las comunas de Cali con alta ocurrencia de denuncia de abuso sexual. Los alcances de esta parte del estudio se presentan a continuación.

\section{MATERIALES Y MÉTODOS}

En el componente cuantitativo del estudio se consideró como fuente primaria de información la base de datos del Centro de Referencia de Medicina Legal y Ciencias Forenses del Suroccidente de Colombia. A partir de ella, se seleccionaron todos los casos de presunto delito sexual, atendidos por clínica y trabajo social forense, ocurridos en el perímetro urbano de la ciudad de Cali, en la población entre 10 a 19 años de edad durante el período 2001-2003. Después, con base en la geo-referenciación se identificó que las comunas 13,14 y 7 , presentaron la mayor prevalencia de casos de denuncia de abuso sexual en población adolescente.

Para el componente cualitativo del estudio, referido a la exploración de la percepción de riesgo de abuso sexual, se definió focalizar las comunas 7 y 13, considerando que las comunas 13 y 14, comparten características socio-demográficas similares y que ambas perte- 


\section{Cuadro 1}

Temas usados para generar la discusión en los grupos focales

\begin{tabular}{ll}
\hline - & Riesgos generales que se perciben en la adolescencia \\
. & Conceptos de abuso sexual \\
\hline - & Percepción de riesgo de abuso sexual: sitios de riesgo, población con mayor riesgo y razones del riesgo \\
. & Razones para que se den los abusos \\
\hline - & Características del abusador \\
\hline
\end{tabular}

necen al Distrito de Aguablanca. Con base en datos del DANE se ubicaron las sedes educativas y sus satélites, donde se realizaron los grupos focales con los estudiantes.

Una vez los centros educativos concedieron la autorización para efectuar el estudio, se solicitó a la coordinación académica de las dos sedes educativas principales, contarles a los estudiantes los objetivos del estudio y motivar su participación. Así, a los estudiantes que de manera voluntaria aceptaron participar en el estudio, se les entregó la forma de consentimiento informado, que debían traer de vuelta con la firma del padre, la madre o el acudiente, en caso que aceptaran la participación del menor de edad en el estudio.

En la fecha prevista para efectuar los grupos focales, previa verificación de la firma del consentimiento informado por parte del adulto responsable del menor, se procedió a explicarles a los estudiantes el consentimiento informado que debían diligenciar. Así, quienes de manera voluntaria ratificaban el deseo de participar en el estudio, procedieron a firmar su propio consentimiento.

Los grupos focales se realizaron teniendo en cuentaque los participantes compartieran características similares de sexo y edad (10-12 años; 13-15 años y 16-19 años), con el fin de facilitar el intercambio de opiniones entre los participantes debido a la afinidad entre pares. Los grupos focales los orientaron dos profesionales del área de salud y de las ciencias sociales, con experiencia en estudios cualitativos, y entrenamiento en el manejo de la técnica de grupo focal así como en el trabajo con población adolescente. Los grupos focales se hicieron en salones de los centros educativos que garantizaban comodidad y privacidad. Se tuvo en cuenta el criterio de saturación de la información para suspender su recolección.

Al iniciar el grupo focal cada estudiante diligenció un formato individual con información referida a edad, sexo, escolaridad, barrio, tiempo de residencia en el barrio y personas con las que convivía. Para promover la discusión en los grupos focales se diseñó una guía de discusión flexible (Cuadro 1).

Se enfatiza que una vez que se exploraba de manera abierta la noción de abuso sexual con cado grupo de adolescentes, luego se procedía a definir este concepto para orientar los ejes de indagación con los que se continuaría la discusión, con el propósito de recuperar la percepción de riesgo específico con respecto a este suceso. El abuso sexual es el que se comete en cualquier tipo de relación entre un adulto y un menor de edad, en la que éste último es obligado a través de la fuerza, la manipulación, el engaño o el soborno a ejecutar o permitir que se den en él conductas sexuales que el ofensor determine. El objetivo del adulto es obtener gratificación sexual. Esto puede ser incluso conductas que no implican contacto físico, por ejemplo: acoso verbal, llamadas telefónicas, mostrar material pornográfico, exhibir los genitales. Como también, puede ser que la relación implique contacto directo, donde el menor de edad puede tener participación activa o pasiva: besos, roces, tocamientos de los genitales o contactos de la boca con los genitales.

Las características de la definición de abuso sexual que orientó la exploración de percepción de riesgo fue: «El abuso sexual se comete en cualquier tipo de relación entre un adulto y un menor de edad, en la que éste último es obligado a través de la fuerza, la manipulación, el engaño o el soborno a ejecutar o permitir que se den en él, las conductas sexuales que el ofensor determine. El objetivo del adulto es obtener gratificación sexual. Esto puede ser incluso conductas que no implican contacto físico, como acoso verbal, llamadas telefónicas, mostrar material pornográfico, exhibir los genitales; también puede ser que la relación implique contacto directo, donde el menor de edad tenga participación activa o pasiva como besos, roces, tocamientos de los genitales o contactos de la boca con los genitales». Esta definición de abuso sexual la elaboró el trabajador social del 
Instituto de Medicina Legal y Ciencias Forenses del Suroccidente. Se resalta que durante el transcurso de cada grupo focal, las investigadoras hicieron referencia reiterada a la definición que orientaba la discusión.

La información proveniente de todos los grupos focales se grabó en audio y después se hizo la trascripción textual. Se llevó a cabo un análisis temático complementado con análisis de red temática ${ }^{1}$, en el cual inicialmente por cada grupo focal se codificó todo el material de acuerdo con las categorías preliminares y emergentes, incluyendo las partes de las transcripciones en cada categoría. Después, se identificaron los temas de cada texto, se refinaron éstos, y se procedió a identificar los temas básicos. Finalmente, se elaboró y se describió la red temática y se comparó la información entre los grupos focales y las respuestas de sus participantes al interior de cada grupo focal, según edad y género.

Una vez terminaba cada grupo focal las investigadoras dieron recomendaciones a los participantes sobre la ruta de atención en caso de sospecha de un caso de acoso o de ser víctima de un abuso sexual. Al finalizar toda la recolección de información, en cada centro educativo se fijó un cartel sobre la ruta de atención propuesta por el Instituto Nacional de Medicina Legal y Ciencias Forenses y el trabajador forense de dicha institución dictó una charla, a docentes y personal directivo, sobre la prevención y la atención del abuso sexual.

Por último, los resultados cuantitativos y cualitativos del estudio se presentaron y discutieron con los docentes y las directivas de los dos centros educativos, con el fin de ser usados en sus procesos educativos. La investigación la aprobó el Comité de Ética de la Universidad del Valle.

\section{RESULTADOS}

En el estudio participaron 121 adolescentes (53 hombres y 68 mujeres) entre los 11 y 19 años de edad, estudiantes de bachillerato de las dos sedes educativas públicas principales de dos comunas de los estratos 1 y 2 de Cali con alta prevalencia de denuncia de abuso sexual en población adolescente. En total se realizaron 10 grupo focales distribuidos en tres franjas de edad y separados por género. Sólo un estudiante no participó en el estudio, pues argumentó que no le gustaba hablar de temas personales con sus compañeros de colegio.
A continuación, se presentan los hallazgos del estudio organizados de acuerdo con los ejes de indagación propuestos.

¿Qué es eso de abuso sexual? Se encontró que los y las adolescentes participantes tienen una noción de abuso sexual aproximada a la socialmente divulgada. Es decir, los conceptos de uso de la fuerza, engaño o amenaza para obtener beneficio o placer genital, sin consentimiento de la otra persona, fueron los más referenciados cuando se les indagó acerca de lo que ellos consideran como abuso sexual.

La noción de abuso sexual centrada en la fuerza se vinculó a la situación de un hombre que con más capacidad física, obliga a mujeres (adultas o adolescentes) a participar de un acto sexual no consentido; por tanto, se asume que éstas son más vulnerables a la violencia sexual por su «fragilidad», con desventaja física frente a los hombres. Este pensamiento que es fuertemente arraigado y mantenido por la cultura, se reproduce en los adolescentes y configura el estereotipo de un victimario masculino y fuerte.

A su vez, la amenaza se relacionó con el uso de la intimidación física o verbal, que conduciría a la persona a acceder a un acto sexual, condición «permisiva» debido al miedo a ser lastimada ella y/o su familia. Al respecto, se señalaron ejemplos, relacionados con el uso de armas para intimidar o de amenazas verbales de matar a personas afectivamente cercanas a la probable víctima, sino accede al acto sexual propuesto.

Por su parte, con el engaño el victimario consigue aprovecharse de la condición de desigualdad en el desarrollo mental o emocional, entre él y su posible víctima, de tal forma que logra que ésta participe en un acto que no quiere y del cual no está consciente, pero al que ha sido llevada mediante distintas tretas. En este caso la referencia fue que «los engañados» siempre eran los niños o las niñas.

\section{«Abuso sexual es forzamiento que hacen los hombres o las mujeres contra un menor. Lo forzan (sic) a tener relaciones con ellos por placer».}

Con lo anterior, se puede concluir que los adolescentes no centran la ocurrencia del abuso sexual sólo en el uso de la violencia física, sino que reconocen otras formas de maniobra de los abusadores.

Por otro lado, los adolescentes mayores, es decir de 
15 años o más, explicitaron que el perpetrador al incurrir en una situación de abuso sexual obtiene beneficio sexual o placer cuando hay penetración, o sea cuando ocurre una violación. En este caso, se señaló que el hecho implicaría a un hombre adulto (victimario) con una mujer o con un menor de edad (víctima), siendo que en esta situación no se consideró la posibilidad de comprometer como víctimas a hombres adolescentes o adultos.

A su vez, los participantes de menor edad (entre 11 y 12 años) fueron más vagos en sus nociones, al referirse a lo que se debe considerar un abuso sexual, pues parecían no tener claridad sobre cómo el victimario obtiene gratificación sexual, más bien se centraron en señalar que es un tipo de sometimiento que causa malestar.

\section{"Entonces, un abuso sexual es algo muy a la fuerza, $y$ algo que una mujer y los niños, es algo que no quieren, es algo que les causa mucha rabia».}

Además, de manera muy tangencial, en la noción de abuso sexual se incluyó el tema del tocamiento o manoseo de las partes íntimas a un niño o niña por parte de un adulto, pero se refirió en relación con la necesidad de saber diferenciar entre las características de las caricias que un adulto da por afecto a un menor de edad, de aquellas que se proporcionan en un contexto de abuso sexual («con morbosidad»).

Por tanto, se expresó que cuando se es un niño pequeño hay situaciones en que es difícil diferenciar de manera inmediata el tipo de caricias que se recibe por parte de los adultos, pero a medida que se van haciendo mayores y se analizan ciertas situaciones es posible que se sea más consciente y se puedan diferenciar el tipo de caricias que se reciben por parte de los adultos.

\section{«(...) sabe que, hay unos familiares que lo acarician a uno, pero son caricias de amor, de afecto, ya. En- tonces, uno tiene que saber diferenciar entre esto yotras personas, que ya lo empiezan a tocar otras partes, las partes íntimas, eso ya es un abuso para mí».}

Esta posibilidad de manoseo no surgió al referirse a los adolescentes o a las mujeres, quizá porque se considera que en estas etapas de la vida la persona tiene capacidad para distinguir entre una caricia que se hace en un marco de una relación afectiva, de aquella que se presenta en una situación de abuso.

Por otro lado, se encontró que todas las nociones de abuso sexual tuvieron como referente lo que han visto o escuchado a través de los medios de comunicación. De hecho, en todos los grupos focales fue recurrente señalar el caso de un abusador de menores de edad que fue ampliamente divulgado por los medios de comunicación en el país. Además, los adolescentes relacionaron la palabra abuso con la frase cotidiana de «abuso de confianza», por tanto, desde su punto de vista el abuso sexual lo comete alguien que es conocido y de esa manera «abusa de la confianza» que en esa persona se ha depositado.

\section{«(...) o sea de personas cercanas a la que es la \\ víctima... eso es un abuso porque se le pierde la confianza, porque nunca pensó que fuera a haceralgoasí».}

Por último, se resalta que hubo consenso en que es más lamentable una «violación» o un «abuso sexual» cuando la mujer no ha iniciado vida sexual. La gravedad del vínculo entre la primera relación sexual con una situación de abuso, se expresó de forma muy emotiva en el grupo de mujeres adolescentes, quienes de manera muy clara expresaron el impacto negativo que podría tener para la vida futura de una joven el ser víctima de abuso sexual, ya que consideran que «perder la virginidad» es un asunto relacionado con un evento romántico, consentido y esperado. Además, se señaló que el mayor riesgo de un abuso es quedar embarazada, pues los padres se enterarían, o contagiarse de una infección de transmisión sexual o de VIH-SIDA.

\section{“(...) más que todo una niña, que todavía es virgen y digamos que es muy inocente sobre estas cosas y llegar una persona abusar de eso y quitarle eso que es lo más preciado para las mujeres y entonces ella crece como con un trauma y ya no sabe qué hacer».}

Llama la atención que las adolescentes en su condición actual de mujer púber frente al temor de ser abusadas sexualmente, se preocupen por el riesgo de 
quedar embarazadas y la relación con el conocimiento que puedan tener los padres sobre el hecho, como si desde su perspectiva el abuso fuera un fenómeno que connotara mayor impacto psicosocial en la familia que en la propia víctima.

Acerca de los escenarios de riesgo sexual de mayor riesgo de abuso sexual. La percepción de los y las adolescentes sobre los escenarios de riesgo para abuso sexual, se focalizó tanto en espacios públicos como privados. En los primeros, se señalaron aquellos sitios en los que se entra en contacto cercano con otras personas adultas y donde se han tenido experiencias propias de intimidación o se ha conocido sobre situaciones de riesgo vividas por otros adolescentes. Estos lugares sólo los indicaron los hombres adolescentes mayores de 15 años de edad.

Además, se expresó que los espacios públicos desolados y en los parques o esquinas donde hay hombres adultos que consumen drogas psicoactivas ilegales, son ambientes propicios para la ocurrencia de abuso sexual. A su vez, la casa de residencia donde se vive con la familia, fue el único espacio privado que se señaló de riesgo.

Las peluquerías, los establecimientos donde se ofrecen servicios de juegos electrónicos en los barrios (las llamadas casas de maquinitas) y los campos de entrenamiento de fútbol, fueron los lugares que se indicaron de alto riesgo para la ocurrencia de abuso sexual para los adolescentes; pues allí, el contacto físico que se hace con el peluquero, el dueño del establecimiento o el entrenador de fútbol, es aprovechado por éstos para seducir sexualmente al adolescente, manosearlo o proponer el intercambio del servicio que se ofrece en el lugar (por ejemplo, el alquiler de una máquina de juegos), si el adolescente accede a una relación con características sexuales.

Se señala que estos lugares de riesgo sólo fueron referenciados por los hombres adolescentes, mientras las mujeres hicieron más énfasis en que el riesgo está en el espacio de la casa y en los lugares públicos desolados o donde hay adultos que consumen drogas.

«(...) porque los peluqueros, le están cortando el pelo y empiezan a tocarlos y si el pela'o le gusta, cierran puertas $y \ldots$...

Adicionalmente, los adolescentes expusieron expe- riencias propias o de sus amigos acontecidas en los tres lugares de riesgo (campo de fútbol, peluquería y establecimiento de alquiler de máquinas electrónicas), allí en diversas oportunidades han recibido ofrecimientos para participar en actos con contenido sexual e incluso han sido objeto de tocamientos de sus partes íntimas o se han visto enfrentados a situaciones en que el adulto ha puesto en contacto sus partes íntimas con sus cuerpos.

A su vez, el espacio doméstico no es percibido como un lugar seguro por las adolescentes, pues temen ser asediadas por algún familiar del sexo masculino y, además, consideran que un hombre extraño puede ingresar a la vivienda, y aprovechar cuando ellas están solas en casa. A su vez, los parques del sector de residencia, donde se congregan hombres a consumir droga, fueron considerados también lugares de riesgo, debido a la relación que establecen los adolescentes que los casos de abuso se presentan porque «el abusadon» está bajo los efectos de narcóticos y no porque dichos lugares sean realmente de riesgo, pues generalmente en ellos hay circulación de personas.

Cuando se han tenido experiencias de riesgo de abuso o de abuso... Al indagar a los y las participantes respecto a las ocasiones en que consideraban que habían sentido o estado amenazado o en riesgo de un abuso sexual, fueron los hombres quienes dieron un mayor número de ejemplos. Las mujeres, por el contrario, no se sintieron cómodas frente a este tipo de indagación.

Las experiencias de riesgo que informaron los hombres adolescentes fueron las relacionadas con la incitación por parte de hombres adultos conocidos a realizar algún acto sexual a cambio de dinero, cosas o actividades, como ingreso a un equipo de fútbol, facilidad para jugar «maquinitas» o acceder a elementos de peluquería. Además, expusieron que en lugares públicos, como las panaderías, es frecuente que otros hombres adultos les toquen las partes íntimas al tiempo que los intimidan con frases obscenas.

Finalmente, al indagar respecto a su manera de reaccionar frente a estas situaciones, la frase típica y reiterada de defensa fue decir al agresor «tráteme serio» y evadirlo dirigiéndose a otro lugar. Todas las experiencias de riesgo de los hombres se enmarcaron en incitarles a actuar, mostrarles partes íntimas o buscar más cercanía física para establecer una relación posiblemente sexual (situaciones de coerción sexual). 
“Otro día fui a la peluquería, y le dije: -Vea, regáleme un poquito de alisadora y me dijo: - Si se la deja chupar y le dije: - Noo, tráteme serio. Vea que no tengo nada ¿Me va a regalar el poquito de alisadora? $y$, entonces, yo me quedé ahí parado y me dijo: - $i A h$ ! Usted la tiene que tener así y le dije no tengo nada y pasó así y llega y me pegó allí, en el pipí».

Con respecto a las mujeres, sólo una joven expresó que en su infancia, sintió que un tío le hacía caricias que iban más allá de lo que ella actualmente considera que se debe permitir en una relación familiar afectiva, y reconoce, por tanto, cuando ya era mayor, que ese acto se podría considerar como una experiencia de abuso sexual.

Razones para tener riesgo de abuso sexual. Las respuestas de los participantes frente al interrogante de porqué hay riesgo de abuso sexual en la adolescencia, muestran que tanto hombres como mujeres tienen actitudes de «culpabilizar» a las mujeres de tener mayor riesgo de abuso sexual, debido a la forma en que a su juicio éstas se comportan y se visten; indicando con ello, que son las mujeres, por tanto, quienes se exponen en mayor medida al riesgo.

En ese sentido, los vestuarios como las faldas y pantalones cortos y las blusas sin mangas y cortas, fueron indicados como los elementos que provocan a los hombres a incurrir en abuso sexual contra las mujeres adolescentes.

"Ah!, cuando uno se pone esos shortcitos y esas

blusitas todas pegaditas y esos yines que le quedan

todos corticos, esos descaderados, eso motiva alos hombres."

"Porque las mujeres andan con sus falditas, mostrando todo lo que tienen...»

A su vez, comportamientos de las adolescentes en la calle considerados como excesivamente extrovertidos frente a los hombres, fueron vistos como elementos de provocación para éstos, que al parecer les incita a violentar a las mujeres. Estos comportamientos son percibidos como «libertinaje», que hacen que las mujeres adolescentes sean quienes tengan más riesgo de abuso sexual.
«Hay veces cuando los hombres están por ahí en las esquinas, las niñas van pasando todas arreboladitas, y excitan a los hombres».

«(...) la pueden ver mucho en la calle, que le gusta la rumba, que las ven besándose en la esquina con uno y otro. También puede ser eso».

Sólo dos mujeres de más de 14 años de edad participantes en uno de los grupos focales, fueron tajantes al expresar que la forma de vestir no se debería considerar como una forma de provocación, pues la mujer debía tener autonomía para llevar la ropa con la que se sienta mejor y concluyeron diciendo que a las jóvenes les gusta la ropa ajustada y corta porque las hace ver atractivas, lo cual no implica que estén provocando ser objeto de abuso sexual.

"iYo les voy a hablar francamente! A mí me gusta que me miren, que me admiren, a mí me gusta que me digan estás bonita. Yo digo que no importa que la mujer se vista tapado o destapado, después que usted tenga su cuerpo bonito y su cara bonita, si se viste tapada, de todas maneras la gente la admira porque se ve bonita».

Además, los hombres adolescentes mayores relacionaron las razones de riesgo con la exposición al riesgo; es decir, el tener malas compañías, el consumo de alcohol y el no seguir las recomendaciones de los padres, hace que sea en la adolescencia donde se tenga más riesgo de un abuso sexual. El relato de un caso de un amigo que bajo los efectos del alcohol fue víctima de abuso sexual, fue el ejemplo usado para ilustrar la exposición a riesgos en esta edad. A su vez, consideran el sentido personal de invulnerabilidad de los jóvenes hace que constantemente estén expuestos a distintos riesgos.

"Porque uno a esta edad cree que lo sabe todo. Por eso, uno no admite muchas veces consejos, como dice... Olvida los consejos que le dan sus padres, cree que con la experiencia que tiene, puede tomar el mundo con una sola mano, y es allí cuando nos estrellamos con el verdadero sentido que tiene la vida. Que uno a los 15 años se cree blindado». 
Por otro lado, al referirse al riesgo que se tiene entre los menores de edad, se consideró que éste se da por descuido de los padres, quienes no previenen suficientemente a sus hijos sobre los riesgos existentes en la vida diaria o los dejan solos en casa de manera reiterada.

¿...Pero quién tiene más riesgo? La percepción sobre quién tiene más riesgo de ser víctima de un abuso sexual, se relacionó con las razones de riesgo. Es decir, los hombres y las mujeres participantes, estuvieron de acuerdo al señalar que son las mujeres quienes tienen mayor riesgo de abuso sexual, lo cual se corresponde con las actitudes pro-abusador, ya mencionadas. Además, consideran que un factor que favorece el riesgo es la condición misma de ser mujer, relacionándola con la debilidad física comparada con la fuerza de los hombres.

En segunda instancia, se informó que los hombres de 13 ó 14 años de edad, también tienen riesgo porque los abusadores gustan de los menores que ya están físicamente formados. Específicamente, al referirse a formados los adolescentes dan cuenta del desarrollo de sus genitales (tamaño del pene) lo cual, a su juicio, incita a los abusadores.

Por último, se señaló que el riesgo también recae sobre los niños o niñas de 7 u 8 años de edad, pues por su ingenuidad pueden fácilmente dejarse engañar de una persona adulta y porque no saben muy bien lo que es abuso sexual.

Creencias respecto al perfil de un abusador. Al indagar de manera general con los adolescentes sobre quién podría ser un abusador, las respuestas de los-las participantes se refirieron rápidamente a considerar a cualquier persona conocida o desconocida, con cualquier apariencia física. Sin embargo, al solicitárseles que describieran de manera más amplia a esta persona, hubo consenso en tres características: sexo masculino, aspecto físico desagradable y adicto a alguna sustancia psicoactiva (alcohol o drogas ilegales). Adicionalmente, consideraron que los abusadores son personas que han sufrido experiencias traumáticas en su infancia y, por tanto, eso les lleva a querer hacer daño a otros, especialmente a menores de edad.

Por otro lado, los adolescentes son categóricos en clasificar a los abusadores sólo como del sexo masculino. Al respecto, los adolescentes hombres mayores de 15 años de edad, expresaron la dificultad de definir una situación de abuso cuando es una mujer mayor quien les propone participar en un acto sexual. Incluso, conside- ran que el hecho de rechazar «la seducción de una mujer» podría poner en cuestión su hombría, tanto ante la misma mujer como con sus pares.

\section{DISCUSIÓN E IMPLICACIONES}

En este estudio mujeres y hombres adolescentes centraron su noción de abuso sexual en torno a la violación (penetración de pene u otro objeto dentro de la vagina o el ano). De hecho, al solicitárseles que definieran que consideraban como abuso sexual, no explicitaron las distintas modalidades de éste, como el tocamiento o la exhibición de las partes íntimas por parte de otra persona. Probablemente esto se deba al énfasis que los medios de comunicación han hecho respecto sólo a las implicaciones jurídicas y morales de una violación o a la percepción que este tipo de abuso sexual sea el que más gravedad presenta. Además, en este estudio se encontró que prevalece el estereotipo del abusador de sexo masculino y las actitudes de culpabilizar a la víctima.

Aunque en la noción de abuso sexual la relación adulto-niño/niña apareció de manera más reiterada, también se consideró que el abuso se puede dar por parte de hombres adultos contra mujeres adultas u hombres adolescentes. Sin embargo, la coerción o la agresión sexual entre adolescentes, que se informa en distintos investigaciones, no fue referenciada entre los adolescentes del estudio, probablemente ello se deba a que no lo perciben como conductas relacionadas con la victimización sexual ${ }^{10}$.

Por tanto, los resultados del estudio indicarían, la necesidad de enfatizar entre las-los adolescentes la gravedad que lleva el abuso sexual cualquiera que sea su modalidad, sin importar el tipo de relación entre víctima y victimario y se destaca la importancia de desmitificar los aspectos relacionados con la percepción que las jóvenes con sus comportamientos o vestuarios auto-inducen ser víctimas de un abuso sexual.

Respecto al estereotipo masculino, otro estudio ya había dicho que tanto en estudiantes adolescentes como en profesionales que atienden casos de abuso sexual prevalece la percepción de abusador masculino heterosexual $^{11}$. Por ello, sería necesario desvirtuar los esquemas que estructuran la visión androcéntrica, en la cual se devalúa lo femenino y se exalta lo masculino, al considerar que lo femenino pertenece a un orden natural de debilidad, carente de fuerza física y mental, con lo 
cual se continúan anclando paradigmas legitimadores de la supremacía masculina, y sobrecargan el concepto que la virilidad la impone el hombre en cualquier circunstan$\operatorname{cia}^{12}$.

Asimismo, entre los adolescentes hombres se requiere reflexionar respecto a las formas de reaccionar frente a los actos de seducción de los que son objeto por parte de mujeres adultas, con lo cual ellos consideran que el hombre al negarse a participar en los actos sexuales propuestos pone en entredicho su masculinidad. Esta actitud refleja las formas como la sociedad culturalmente establece modos de inserción de cada sexo en la realidad, que al éstos ser transformados o modelados cuestionan el envoltorio ideológico de la persona, en este caso al sexo masculino, al verse cuestionada su virilidad ${ }^{13}$.

Por otro lado, las actitudes de culpabilizar a la víctima se sustentan en los mitos que son cierto tipo de mujeres las que pueden ser víctimas de abuso sexual ${ }^{14}$.También, según lo plantea Bordieu, la diferenciación de roles y usos (prótesis) del cuerpo marcan señales culturalmente asimiladas en los hombres para colocar a la mujer como objeto de deseo, signos y símbolos equivocadamente construidos que estimulan prácticas arbitrarias hacia el género contrario ${ }^{12}$.

Además, lo preocupante es que históricamente se han argumentado supuestos consentimientos de la persona víctima de violencia sexual, certidumbre de la permisividad, que la culpabiliza de su participación, situación que engendra enmascaramiento de conductas $\mathrm{y}$ actos violentos sexuales, en términos jurídicos y sociales. Este fenómeno de violencia además de ser anodina, repercute en la víctima susceptible de ser invisibilizada y estigmatizada moral y socialmente ${ }^{15}$.

En este estudio, también, se encontraron diferencias de género en la percepción de riesgo de abuso sexual. Los hombres perciben más riesgo en los lugares públicos y, en coherencia con ello, las experiencias de riesgo y de posible abuso las han vivenciado en lugares públicos.

A su vez, las mujeres perciben mayor riesgo en sus casas, aunque no dieron cuenta de experiencias de riesgo, se podría colegir que dicha percepción de riesgo en la esfera doméstica se podría referir a relaciones incestuosas de facto o posiblemente percibidas, si se tiene en cuenta que distintos estudios muestran que hay mayor susceptibilidad a la violencia sexual cuando las características sociodemográficas de las víctimas corresponden a hacinamiento, pobreza, bajos ingresos, familias re-constituidas, monoparentales o mixtas, roles violentos y donde el cuidado de los hijos-as recae en buena parte en los infantes y adolescentes ${ }^{16}$.

El hecho que las mujeres no accedieran a contar sus propias experiencias de riesgo sexual, puede depender a diferencias culturales (tabúes y miedos) en aceptar dicha situación o con la forma directa y grupal cómo se abordó el asunto. Al respecto, otros estudios han mostrado diferencias en la comunicación de experiencias de violencia de acuerdo con las estrategias de recolección usadas (informe cara-cara, informe auto-diligenciado escrito o asistido por computador $)^{17}$.

También, cabe resaltar que la literatura muestra que en comparación con los hombres, son las mujeres de todas las edades quienes manifiestan haber sido víctimas de abuso sexual por personas conocidas o por figuras paternas, lo cual podría ser un indicador que el espacio doméstico (la casa) sea el lugar de más riesgo $\mathrm{y}$, por tanto, las adolescentes en este estudio hayan manifestado su mayor percepción de riesgo en dicho lugar.

Lo anterior supone que el vínculo de consanguinidad no es necesariamente un factor protector para los hijosas, como tampoco el parentesco por afinidad, haciendo vulnerables a las personas que aún requieren de la protección de los adultos. Precisamente, el incesto es una de las prácticas de violencia sexual más antigua y reconocida socialmente en donde el padre biológico o el padrastro son las personas transgresoras de las prescripciones culturales y normativas, y causan confusiones que perturban psicoafectivamente a las víctimas y desmoronan las relaciones parentales ${ }^{18}$.

Por otro lado, cabe destacar que la figura autoritaria de un adulto al ser situada como una construcción social, crea confusión -sustancialmente- en edades tempranas al legitimarse como un dispositivo de credibilidad y confianza de la naturaleza de los lazos paternos, dentro de los roles asignados para el ejercicio de la paternidad legal, social y valorativa de las relaciones institucionales como la familia, y da lugar a relaciones abusivas ${ }^{19}$.

Por último, especial atención merece el informe de los adolescentes, que expresaron haberse visto envueltos en situaciones que ellos perciben de riesgo, cuando en realidad son de abuso sexual, donde su reacción fue sólo decir «tráteme serio», que indica la falta de 
habilidad para actuar frente a la agresión sexual o quizás de conocimiento que les permita diferenciar con claridad la agresión verbal o física de las relaciones o vínculos positivos que se gestan entre las personas.

En coherencia con lo anterior, el estudio de Del $\mathrm{Campo}^{20}$ mostró que frente a una situación de abuso, donde media la amenaza, $25 \%$ de los menores de edad expusieron que cederían y $20 \%$ que aceptarían el soborno de los agresores para guardar silencio frente al abuso, lo cual muestra la poca capacidad de los menores para enfrentar una situación de abuso.

Los resultados de este estudio de percepción de abuso sexual en población adolescentes podrían contribuir a fortalecer las iniciativas de prevención que se diseñen para y con este grupo poblacional. En especial en aquellos aspectos relacionados con la identificación o reconocimiento de situaciones de riesgo o de abuso sexual cualquiera que sea su modalidad, la eliminación de los estereotipos relacionados con abusadores masculinos, principalmente extraños, y con las actitudes de culpabilizar a la víctima.

Conflicto de intereses. Los autores declaran que no hay conflicto de intereses en el presente manuscrito.

\section{REFERENCIAS}

1. OMS. Informe mundial sobre la violencia y la salud. Washington DC: OMS; 2002.

2. Johnson C. Child sexual abuse. Lancet. 2004; 364: 462-70.

3. Senn T, Carey M, Vanable P, Coury-Doniger P, Urban M. Characteristics of sexual abuse in childhood and adolescence influence sexual risk behavior in adulthood.Arch Sex Behav. 2007; 36:637-45.

4. Cloutier S, Martin SL, Poole C. Sexual assault among North Carolina women: prevalence and health risk factors. JEpidemiol Community Health. 2002; 56:265-71.

5. Claramunt MC. Abuso sexual en mujeres adolescentes. Pro- grama mujer, saludy desarrollo serie género y salud pública. San José: OPS/OMS; 2000.

6. Sugar NF, Fine DN, Eckert LO. Physical injury after sexual assault: Findings of a large case series.Am J Obstet Gynecol. 2004; 190: 71-6.

7. Instituto Nacional de Medicina Legal y Ciencias Forenses. Forensis: Datos para la vida 2005. Bogotá, DC: Instituto Nacional de Medicina Legal y Ciencias Forenses.

8. Aguilar A, Salcedo M. Caracterización de la violencia sexual en adolescentes de 10 a 19 años, 2001-2003, Cali.ColombMed. 2008; 39:356-63.

9. Attride-Stirling J. Thematic network: an analytic tool for quality research. QualityRes. 2001; 1:385-405.

10. Oswald DL, Russell BL. Perceptions of sexual coercion in heterosexual dating relationships: the role of aggressor gender and tactics. J Sex Res. 2006; 43:87-95.

11. Fuselier DA, Durham RL, Wurtele SK. The child sexual abuser: perceptions of college students and professionals. SexAbuse. 2002; 4:271-80.

12. Bourdieu P. La dominación masculina. $2^{\mathrm{a}}$ ed. Barcelona: Editorial Anagrama; 2000

13. Amorós C. Hacia una crítica de la razón patriarcal. $2^{\mathrm{a}}$ ed. Barcelona: Editorial Anthropos; 1991.

14. Sheldon JP, Parent SL. Clergy's attitudes and attributions of blame toward female rape victims. Violence Against Women 2002; 8:233-56.

15. Vigarello G. Historia de la violación, siglos XVI-XX. Madrid: Ediciones Cátedra; 1999.

16. Departamento Nacional de Planeación, Banco Interamericano de Desarrollo, Universidad de los Andes. Violencia en las familias colombianas. Costos socioeconómicos, causas yefectos. Bogotá: Gente Nueva Editorial Ltda; 2003.

17. MacMillan HL, Wathen N, Jamieson E, Boyle M, McNutt LA, Worster A, etal. Approaches to screening for intimate partner violence in health care settings. A randomized trial. JAMA. 2006; 296:530-6.

18. Robin Fox. La roja lámpara del incesto. $2^{\mathrm{a}}$ ed. México, DC: Fondo de Cultura Económica; 1990.

19. Bentovim A. Sistemas organizados por traumas: El abuso físico y sexual en las familias. Buenos Aires: Editorial Paidós; 2001.

20. Del Campo Sánchez A. Conhecimentos e atitudes dos pais, menores e professores em relação ao abuso sexual. Análise Psicológica. 2001; 2:253-9. 